\title{
Efficacy of a novel membrane comprising a copolymer of L-lactic acid and glycolic acid in osteoblasts in vitro
}

\author{
Hiroki KUWAHARA, Noriko TACHIKAWA, Kazuhiro KON and Shohei KASUGAI \\ Department of Oral Implantology and Regenerative Dental Medicine, Tokyo Medical and Dental University, Tokyo, Japan \\ Corresponding author, Hiroki KUWAHARA; E-mail: kuwairm@tmd.ac.jp
}

\begin{abstract}
In guided bone regeneration (GBR), a barrier membrane is applied to provide a space for bone regeneration to promote cell adhesion, cell proliferation, and bone regeneration by inducing cell migration to the surface of the bone-deficient site. We prepared a non-woven absorbable membrane for GBR comprised of a copolymer of L-lactic acid and glycolic acid (PLLGA) using the dry spinning method. A thin and dense membrane comprising unsintered hydroxyapatite (uHA) and poly-L-lactic acid (PLLA) was used for comparison. Osteoblast TMS-12 cells were seeded onto the membranes, and cell adhesion and proliferation rates were examined. Calcification levels were quantified by Alizarin Red S staining after cell culture in calcification induction medium. Osteoblastic gene expression was examined by RT-PCR. Cell adhesion and proliferation rates and calcification on PLLGA membranes were significantly higher than those on uHA/PLLA membranes. PLLGA membranes upregulated osteogenic gene expression compared to on uHA/PLLA membranes. Non-woven PLLGA membranes may be useful in GBR for promoting bone formation and maturation.
\end{abstract}

Keywords: Guided bone regeneration, Dental implant, L-lactic acid, Osteoblast

\section{INTRODUCTION}

Prosthetic treatments involving dental implants for patients who are partially or fully edentulous are clinically effective and widely used. However, dental implant treatments require sufficient bone to accommodate the implants. Many cases require bone augmentation because of insufficient amounts of bone. Guided bone regeneration (GBR) is a well-known surgical technique for bone augmentation, in which a barrier membrane is applied to provide a space for bone regeneration. The membrane promotes cell adhesion, enhances cell proliferation, and promotes bone regeneration by inducing cell migration to the surface of the bone-deficient $\operatorname{site}^{1-3)}$. Since the introduction of this method in the late 1980s, various new and improved materials have become available and used to enhance the effectiveness of the procedure ${ }^{4-77}$.

Non-absorbing expandable polytetrafluoroethylene (e-PTFE) was introduced as the first-generation membrane. However, the membrane must be removed surgically, increasing the invasiveness, treatment cost, and risk of postoperative bacterial infections ${ }^{8-13)}$. Thus, resorbable GBR membranes are more desirable. Recently, various synthetic or natural absorbable membranes were developed. Most commonly used absorbable membranes are produced from porcine or bovine-derived collagen ${ }^{7,14-19)}$ and have displayed similar clinical results as e-PTFE without requiring surgical membrane removal. However, the risk of infection of known or unknown diseases in domestic animals cannot be completely eliminated. Additionally, undesirable immune responses to heterologous proteins may occur

Color figures can be viewed in the online issue, which is available at J-STAGE.

Received Oct 8, 2020: Accepted Jan 27, 2021

doi:10.4012/dmj.2020-369 JOI JST.JSTAGE/dmj/2020-369 with these membranes ${ }^{20-22)}$.

Poly(L-lactic acid) (PLLA) and poly(lactic acidco-glycolic acid) (PLGA) are synthetic biodegradable polymers used to prepare biodegradable medical devices ${ }^{23,24)}$. Their application as biomedicals material has been extensively studied to assess materials for bone substitution, tissue engineering, and drug delivery. Indeed, PLLA consists entirely of polymerized L-lactic acid, which does not trigger the infections and immune responses characteristic of animal-derived biomaterial ${ }^{25)}$

A biocompatible and biodegradable bone fixation device (Super-Fixsorb-MX, Teijin Medical Technologies, Osaka, Japan) composed of a composite of unsintered hydroxyapatite (uHA) particles and PLLA was previously developed and clinically applied ${ }^{25,26)}$. This material exhibits a mesh-like structure and end elasticity similar to that of natural cortical bone tissue. This material is robust during bone healing and superior to unfilled PLLA.

Furthermore, it shows optimal degradation and resorption properties, bone conduction, and bone binding capacity $^{27,28)}$. Moreover, hydrophilization treatment of this material further improves its properties ${ }^{29,30)}$.

Numerous studies have reported the usefulness of membranes capable of nonspecific hydrolysis in vivo by polymerizing lactic acid and glycolic acid. Furthermore, although animal experiments have reported the effectiveness of membranes comprising a composite of unsintered hydroxyapatite and PLLA, few related in vitro studies have been performed. So far, our research group has developed membranes used in the GBR method for many years, but they were mainly flat platelike structures. However, bioabsorbable membranes that have recently appeared have a non-woven fabric- 
like structure. Therefore, we developed a non-woven membrane composed of a copolymer of PLLA and poly(glycolic acid) (PGA) using the dry spinning method that exhibits a 3-D structure that is not derived from animals. In this study, we considered that the developed membrane would contribute significantly to cell-cell communication and analyzed in vitro. Here, we developed a non-woven membrane composed of a copolymer of PLLA and PGA using the dry spinning method. This study was conducted to investigate the effects of this membrane on osteoblast adhesion, proliferation, and differentiation.

\section{MATERIALS AND METHODS}

\section{Sample preparation}

L-lactide/glycolide (PLLGA) (82/18 mol\%) membranes of 400-450- $\mu \mathrm{m}$ thickness were prepared by the dry spinning method $^{31)}$. The membranes were trimmed into circles 58 $\mathrm{mm}$ in diameter. These membranes were sterilized with an electron beam. Scanning electron micrographs of the membrane are shown in Fig. 1. This membrane was not porous, and no differences were observed between the properties of the upper and lateral surfaces.

uHA/PLLA dense membranes $20 \mathrm{~mm}$ in diameter and $0.5 \mathrm{~mm}$ in thickness were prepared by a rolling method using material from biodegradable bone fixation devices (Super Fixsorb MX30, Teijin Medical Technologies) composed of $30 \mathrm{wt} \% \mathrm{uHA}$ and $70 \mathrm{wt} \%$ PLLA, as we reported previously ${ }^{25)}$. Scanning electron micrographs of the membranes are presented in Fig. 1. The membranes were not subjected to hydrophilization to alter the surface texture.

All membranes were kindly provided by Teijin
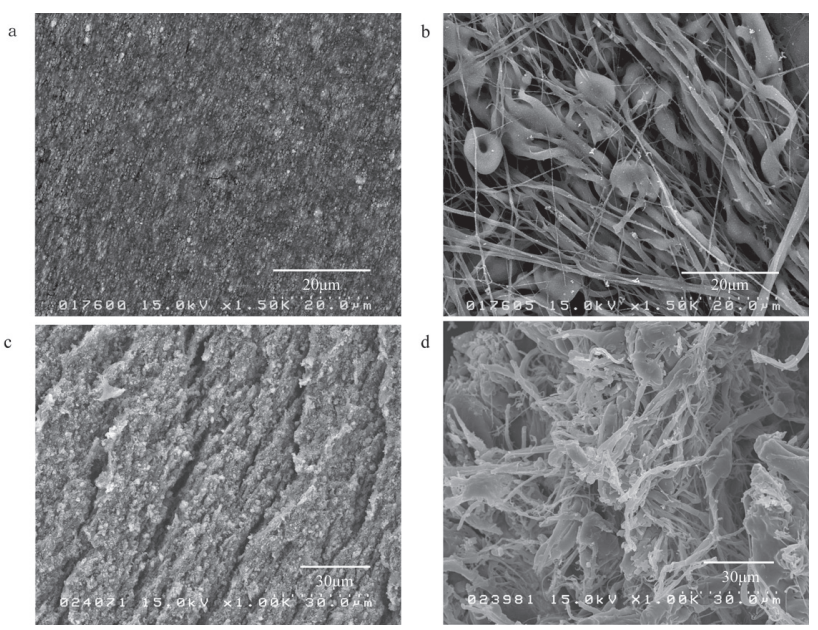

Fig. 1 SEM images.

a) uHA/PLLA membrane: unsintered hydroxyapatite/poly(L-lactic acid), b) PLLGA membrane upper surface view SEM images, c) uHA/PLLA membrane: unsintered hydroxyapatite/poly(L-lactic acid), d) PLLGA membrane lateral surface view SEM images.
(Tokyo, Japan).

Cell culture

TMS-12 cells, previously characterized as murine osteoblasts, were isolated from bone marrow-derived stromal cell ${ }^{32)}$ and cultured in alpha-modified Eagle's medium ( $\alpha$-MEM, Gibco, Grand Island, NY, USA) supplemented with $10 \%$ fetal bovine serum (HyClone, Logan, UT, USA) in $100-\mathrm{mm}$ dishes at $37^{\circ} \mathrm{C}, 100 \%$ humidity, and $5 \% \mathrm{CO}_{2}$.

\section{Cell adhesion and proliferation assay}

Cell adhesion and proliferation were assessed by measuring cell viability with a WST-8-based colorimetry kit (Cell Counting Kit-8, Dojindo Molecular Technologies, Kumamoto, Japan) in accordance with the manufacturer's instructions. This method reveals mitochondrial dehydrogenase activity in living cells by measuring the conversion of WST-8 into a water-soluble disulfonated tetrazolium salt.

To evaluate cell adhesion, TMS-12 cells were seeded into a 12 -well plate on the membranes at a density of $1 \AA \sim 105$ cells/mL and cultured for $1,3,6$, and $12 \mathrm{~h}$.

Cell proliferation was assessed by allowing TMS-12 cells at $1 \AA \sim 104$ cells $/ \mathrm{mL}$ to attach to the membranes, after which culture was terminated at $1,3,5$, and 7 days.

The medium in both plates was replaced with fresh medium supplemented with CCK-8 and 1:10 of the amount of medium in each well and incubated for 1 h. Next, $100 \mu \mathrm{L}$ of the suspension from each well was placed in triplicate in a 96-well plate, and absorbance was measured at $450 \mathrm{~nm}$ for $1 \mathrm{~s}$ (Wallac 1420 ARVO MX, PerkinElmer, Waltham, MA, USA). In the cell proliferation assay, after measurement, the wells were washed several times with warm phosphate-buffered saline, and the cell cultures were supplemented with $\alpha$-MEM.

\section{Alizarin Red S staining}

Alizarin Red staining was performed to assess extracellular matrix mineralization. TMS-12 cells were seeded into a 12 -well plate at $3 \AA ̊ \sim 105$ cells/well on either the uHA/PLLA membranes or PLLGA membranes. For further comparison, we prepared a group without membranes. At 1 day after cell seeding, calcification induction medium containing ascorbic acid $(50 \mu \mathrm{g} / \mathrm{mL}$, Nacalai Tesque, Kyoto, Japan) and $\beta$-glycerophosphate (5 mM, Sigma, St. Louis, MO, USA) was added to each well, and the culture medium was changed once every 1-2 days. After 7 days, the cells were fixed in $75 \%$ ethanol for $1 \mathrm{~h}$ and stained with Alizarin Red in water $(40 \mathrm{mM}, \mathrm{pH}$ 6.4) at room temperature for $5 \mathrm{~min}$. Extraction was carried out for 20 min using cetylpyridinium chloride (SigmaAldrich), and absorbance was spectrophotometrically measured at $560 \mathrm{~nm}$ as previously reported. Furthermore, under scanning electron microscopy, bone nodules were observed on the membrane. 


\section{Real-time RT-PCR analysis}

After dividing the cells into groups with and without membranes, TMS-12 cells were seeded at a density of 10,000 cells per $\mathrm{mL}$ cell culture dish. The group with the membrane contained cells seeded on PLLGA membranes with a 58-mm diameter. One day after cell seeding, the cells were cultured in calcification induction medium, and the medium was changed every other day. After 1, 3, and 5 days of culture, total RNA from the cells was isolated using TRIzol reagent (Thermo Fisher Scientific, Waltham, MA, USA) in accordance with the manufacturer's instructions. Five micrograms of total RNA were subjected to reversetranscription (ReveTra Ace, TOYOBO, Osaka, Japan). cDNA samples were amplified using a KAPA SYBR Fast qPCR kit (KAPA BIOSYSTEMS, Wilmington, MA, USA) under the following cycling conditions: $95^{\circ} \mathrm{C}$ for $3 \mathrm{~min}$, followed by 40 cycles at $95^{\circ} \mathrm{C}$ for $3 \mathrm{~s}$ and $60^{\circ} \mathrm{C}$ for $45 \mathrm{~s}$. Amplifications were performed on an ABI 7500 Real-time PCR system (Applied Biosystems, Foster City, CA, USA). The gene-specific primers were as follows: ALP (forward, GAGCGTAATCTACCATGGAG) and (reverse, CTTGACTGTGGTTACTGCTG); BSP (forward, TACCGGCCACGCTACTTTCTTTA) and (reverse, GACCGCCAGCTCGTTTTCATCC); OCN (forward, CCGGGAGCAGTGTGAGCTTA) and (reverse, TAGATGCGTTTGTAGGCGGTC); Gapdh, (forward, GCCTGGTCCCGTAGACAAAA) and (reverse, TAGGGCCTCTCTTGCTCAGT).

\section{Statistical analysis}

Numerical data are presented as the mean \pm standard deviation. Data were analyzed using a two-tailed Student $t$ test to compare two groups and Tukey-Kramer method for multiple comparisons (JMP14, SAS Institute, Cary, NC, USA). $p<0.05$ was considered to indicate a significant difference.

\section{RESULTS}

Observation by SEM to investigate the surface properties of the membrane revealed a structure in which the nonwoven fiber was three-dimensionally entangled in the PLLGA membrane. In contrast, a plate-like structure was observed for the uHA/PLLA membrane (Fig. 1).

The cell adhesion rate of TMS-12 cells to the PLLGA membrane was significantly increased at $6 \mathrm{~h}$ after cell seeding compared to on the uHA/PLLA membranes (Fig. 2a). Furthermore, the cell proliferation rate on the PLLGA membrane was significantly higher than that on the uHA/PLLA membrane after 3 days of culture. The number of cells on the PLLGA membrane continuously increased, whereas the increase in cells on the uHA/ PLLA membrane or in the culture plate was slow and almost reached a plateau early (Fig. 2b). Calcification was quantified by Alizarin Red S staining on day 7 of cell culture in calcification induction medium. The amount of calcification was significantly higher on the PLGA membrane than on the UHA/PLLA membrane (Fig. 3).

Mineralization of TMS-12 cells was observed under a stereoscopic microscope after culturing the cells in calcification induction medium. SEM images of cells on the membranes are shown in Fig. 4. Many elongated cells adhered well to the PLLGA membrane and were multilayered, whereas cells on the uHA/PLLA membrane were flat (Fig. 4).

As shown in Fig. 5, although not significant, there was a tendency of increased expression of the three osteogenic markers in the cells on the uHA/PLLA membrane compared to those on the PLLGA membrane at days 1 and 3. However, this tendency was reversed at day 5. At day 5, the gene expression level of ALP on the PLLGA membrane was higher than the control groups. At day 5, the other two osteogenic markers were also highly expressed in cells on the PLLGA membrane (a)

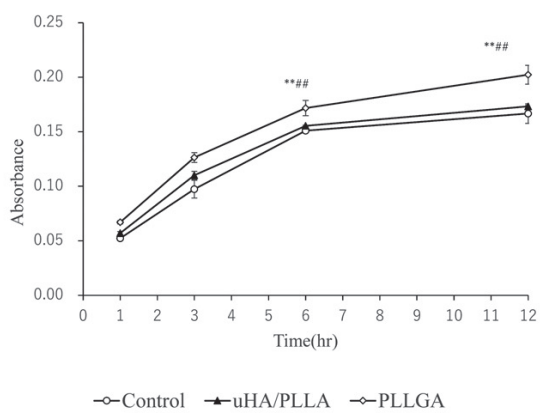

(b)

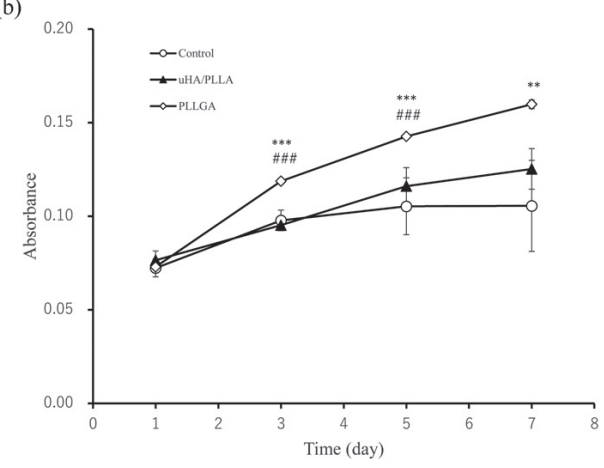

Fig. 2 Cell adhesion and proliferation assay.

(a) Cell adhesion assay. Enumeration of TMS-12 cells after 1, 3, 6, and $12 \mathrm{~h}$ via the CCK-8 assay. ${ }^{*}$ Significantly different from control $\left({ }^{*} p<0.05,{ }^{* *} p<0.01\right)$, \#Significantly different from uHA/PLLA (\#\#p<0.01), Tukey-Kramer's multiple range test. (b) Cell proliferation assay. Enumeration of TMS-12 cells on day 1, 3, 5 , and 7 using the CCK- 8 assay. ${ }^{*}$ Significantly different from control $(* * p<0.01$, $* * * p<0.001)$, \#:Significantly different from uHA/PLLA (\#\#\#p<0.001), TukeyKramer's multiple range test. 


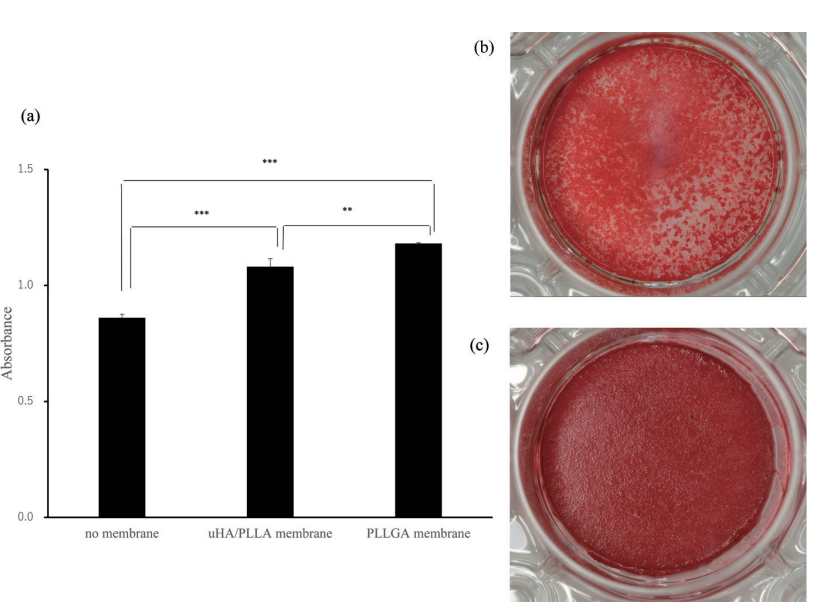

Fig. 3 (a) Quantitative calcification assay. Calcification volume on day 7 after the induction of calcification. Data are presented as mean \pm S.D., $n=3$. TurkeyKramer test. (b) Day 7 after the induction of calcification showing a stained image of the PLLGA membrane. (c) Day 7 after the induction of calcification showing a stained image of the uHA/ PLLA membrane.
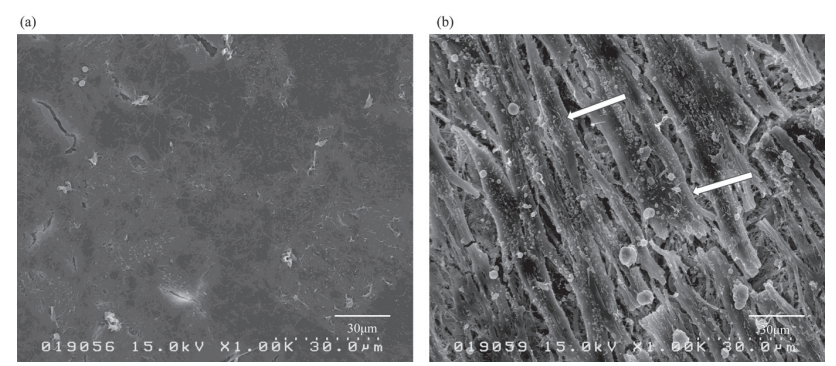

Fig. 4 SEM image after 7 day of culturing in calcified differentiation induction medium.

(a) uHA/PLLA membrane: unsintered hydroxyapatite/poly(L-lactic acid). SEM images of uHA/PLLA membrane showed calcified flat tissue. (b) PLLGA membrane. White arrows: calcified nodules were observed. compared to cells on the uHA/PLLA membrane, although the difference was not significant.

\section{DISCUSSION}

GBR is based on the concept of securing space and facilitating bone regeneration using a membrane. The use of membranes in this study provided an enclosed space, enabling bone regeneration rather than internal growth of connective tissue during bone regeneration ${ }^{7}$. During bone regeneration, the barrier membrane provides a sealed space to facilitate bone regeneration rather than internal growth of connective tissue ${ }^{30)}$; i.e., the membrane material requires sufficient mechanical strength $^{25,31)}$. There are also research reports that porous scaffolds based on microfibers allow adhesion, spreading, orientation and proliferation of cells ${ }^{33)}$. We considered that the three-dimensional morphology was more effective for bone formation that the chemical composition of the membrane.

In this study, a PLLGA non-woven membrane for GBR was investigated using mouse-derived osteoblastlike TMS-12 cells. The osteogenic potential of this material was evaluated in vitro.

It is well-known that $\mathrm{HA}$ is an osteoconductive material. Furthermore, addition of uHA can increase the mechanical strength of PLLA compared to PLLA alone ${ }^{29)}$. As the effectiveness of uHA/PLLA as a bone fixation device has been already reported in animal experiments and clinically, a membrane composed of similar material may be useful for GBR. However, uHA/ PLLA membranes have not been evaluated in vivo or in vitro. Thus, in this study, in addition to using a PLLGA non-woven membrane, we used an uHA/PLLA membrane, which was prepared using a rolling method from the material similar to the resorbable bone fixation device.

We predicted that the UHA/PLLA membrane would stimulate cell attachment and proliferation as well as osteogenic differentiation compared to the PLLGA nonwoven membrane. However, the experimental results (a)

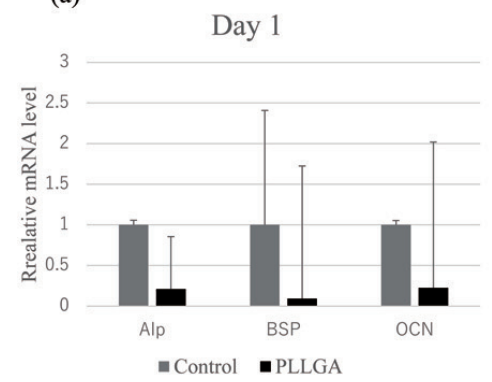

(b)

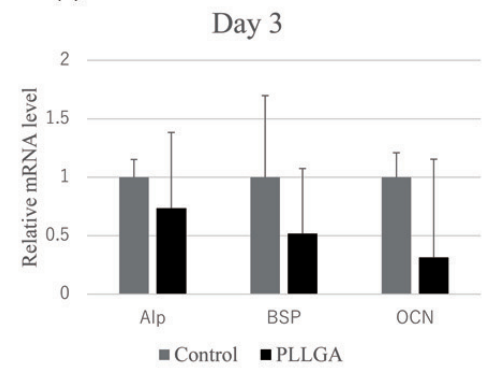

(c)

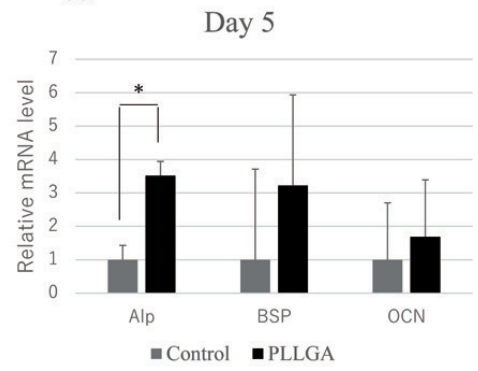

Fig. 5 Osteogenic gene expression levels in TMS-12 cells cultured in calcification induction medium. Expression of osteogenic genes was measured via RT-qPCR analysis at the following time points: (a) day 1 ; (b) day 3 ; (c) day $5(n=3) . * p=0.049$. On day 1 and day 3 , osteogenic gene expression levels were lower in the experimental groups than in the control groups. On day 5, ALP expression levels were significantly higher in the experimental groups. 
differed from our predictions.

In the present study, the promotion of both cell attachment and proliferation were greater on the PLLGA non-woven membranes than on the uHA/PLLA membrane. Furthermore, osteogenic differentiation and mineralization were stimulated on the PLLGA non-woven membrane compared to on the uHA/PLLA membrane.

Cells initially attach to the material surface, followed by cell proliferation. A material that stimulates both cell attachment and proliferation may also promote cell differentiation. In the present study, cell attachment and proliferation together with osteogenic differentiation were more stimulated on the PLLGA nonwoven membrane than on the uHA/PLLA membrane, supporting this speculation. In the cell proliferation assay (Fig. 2b), when the same number of cells was seeded, the control was cultured in a plane, so that it became confluent in day 3 , the proliferation stopped, and the stage of differentiation was entered. In the experimental group of PLLGA membrane, the structure of the material is non-woven fabric and cells proliferate three-dimensionally, so it is considered that the cells are in the proliferative phase even after day 3 .

The osteogenic differentiation markers examined in the present study have been frequently used in previous studies. Alkaline phosphatase is a dephosphorylation enzyme with an important role in mineralization. Bone sialoprotein and osteocalcin are non-collagenous matrix proteins in bone; however, the actual roles of these matrix proteins in bone are unclear. We also examined mineralization, which is considered as the final product of osteogenic differentiation.

Although not significant, the expression of these osteogenic markers on control group was higher than on the PLLGA non-woven membrane at days 2 and 3. However, the expression levels of these osteogenic markers were reversed on day 5 . At least at the stage of day 3 , the experimental group is still in the proliferative phase, so the differentiation is not so advanced. At this point, the control group is already in the differentiation stage, so it is considered that the differentiation marker is highly expressed. On day 5, the cell proliferation of the experimental group almost reached plateau, and the differentiation marker increased. In addition, mineralization on the PLLGA non-woven membrane was more prominent than on the control group. According to the analysis results of RT-qPCR, the markers whose expression is increased are higher in the order of Alp, $\mathrm{BSP}, \mathrm{OCN}$, so it is considered that BSP and OCN will be further increased as the differentiation progresses. Thus, the PLLGA non-woven membrane may promote osteogenic differentiation compared to the uHA/PLLA membrane. The reason is that the PLLGA membrane has a non-woven fabric-like structure, which enables ternary culture and promotes spheroid-like proliferation and calcification of TMS- 12 cells.

Both PLLA and PLLGA are hydrophobic, which is unfavorable for cell attachment and proliferation. We did not treat the membranes using methods that increased their hydrophilicity. uHA is highly hydrophilic; however, although the uHA/PLLA membrane contains uHA, most uHA existed inside the material rather than being exposed on the material surface. Thus, we addition of uHA likely did not increase the hydrophilicity of the uHA/PLLA membrane used in this study.

Notably, as revealed in the SEM images in Fig. 1, the PLLGA non-woven membrane exhibited a threedimensional nonwoven fabric-like structure, whereas the UHA/PLLA membrane was unstructured and dense, forming a thin plate with less space. The difference in the 3-D structure between the two membranes was predicted to influence cell attachment and proliferation, further resulting in differences between osteogenic differentiation and mineralization.

In conclusion, this in vitro study demonstrated the potential of the PLLGA non-woven membrane as a GBR membrane. We previously reported that the PLLA nonwoven membrane, which is similar to presently used membranes, is effective in bone augmentation of the maxillary sinus of rabbits ${ }^{34)}$, supporting our conclusions. However, further animal studies are required to confirm the usefulness of the PLLGA non-woven membrane for GBR.

\section{ACKNOWLEDGMENTS}

This study was supported by Teijin Ltd. The authors have no conflicts of interest to declare. No funding was received for this study.

\section{CONFLICT OF INTEREST}

Hiroki Kuwahara, Noriko Tachikawa, Kazuhiro Kon and Shohei Kasugai have no conflict of interest with this article.

\section{REFERENCES}

1) Lang NP, Hämmerle CH, Brägger U, Lehmann B, Nyman SR. Guided tissue regeneration in jawbone defects prior to implant placement. Clin Oral Implants Res 1994; 5: 92-97.

2) Buser D, Brägger U, Lang NP, Nyman S. Regeneration and enlargement of jaw bone using guided tissue regeneration. Clin Oral Implants Res 1990; 1: 22-32.

3) Nyman S, Lang NP, Buser D, Bragger U. Bone regeneration adjacent to titanium dental implants using guided tissue regeneration: A report of two cases. Int J Oral Maxillofac Implants 1990; 5: 9-14.

4) Watzinger F, Luksch J, Millesi W, Schopper C, Neugebauer $\mathrm{J}$, Moser D, et al. Guided bone regeneration with titanium membranes: A clinical study. Br J Oral Maxillofac Surg 2000; 38: $312-315$.

5) Urban IA, Jovanovic SA, Lozada JL. Vertical ridge augmentation using guided bone regeneration (GBR) in three clinical scenarios prior to implant placement: a retrospective study of 35 patients 12 to 72 months after loading. Int J Oral Maxillofac Implants 2009; 24: 502-510.

6) Chiapasco M, Romeo E, Casentini P, Rimondini L. Alveolar distraction osteogenesis vs. vertical guided bone regeneration for the correction of vertically deficient edentulous ridges: A 1-3-year prospective study on humans. Clin Oral Implants Res 2004; 15: 82-95. 
7) von Arx T, Buser D. Horizontal ridge augmentation using autogenous block grafts and the guided bone regeneration technique with collagen membranes: a clinical study with 42 patients. Clin Oral Implants Res 2006; 17: 359-366.

8) Schenk RK, Buser D, Hardwick WR, Dahlin C. Healing pattern of bone regeneration in membrane-protected defects: A histologic study in the canine mandible. Int J Oral Maxillofac Implants 1994; 9: 13-29.

9) Takata T, Wang HL, Miyauchi M. Migration of osteoblastic cells on various guided bone regeneration membranes. Clin Oral Implants Res 2001; 12: 332-338.

10) Wang HL, Miyauchi M, Takata T. Initial attachment of osteoblasts to various guided bone regeneration membranes: An in vitro study. J Periodontal Res 2002; 37: 340-344.

11) Chiapasco M, Abati S, Romeo E, Vogel G. Clinical outcome of autogenous bone blocks or guided bone regeneration with e-PTFE membranes for the reconstruction of narrow edentulous ridges. Clin Oral Implants Res 1999; 10: 278288.

12) Lindfors LT, Tervonen EA, Sándor GK, Ylikontiola LP. Guided bone regeneration using a titanium-reinforced ePTFE membrane and particulate autogenous bone: the effect of smoking and membrane exposure. Oral Surg Oral Med Oral Pathol Oral Radiol Endod 2010; 109: 825-830.

13) Dies F, Etienne D, Abboud NB, Ouhayoun JP. Bone regeneration in extraction sites after immediate placement of an e-PTFE membrane with or without a biomaterial. A report on 12 consecutive cases. Clin Oral Implants Res 1996; 7: 277 285.

14) Parodi R, Carusi G, Santarelli G, Nanni F. Implant placement in large edentulous ridges expanded by GBR using a bioresorbable collagen membrane. Int J Periodontics Restorative Dent 1998; 18: 266-275.

15) Zahedi S, Legrand R, Brunel G, Albert A, Dewé W, Coumans $\mathrm{B}$, et al. Evaluation of a diphenylphosphorylazidecrosslin ked collagen membrane for guided bone regeneration in mandibular defects in rats. J Periodontol 1998; 69: 12381246 .

16) Geistlich P, Zdenek E, Philip B. Resorbable collagen membrane for use in guided tissue regeneration. U.S. Patent No. 5,837,278. 17 Nov. 1998.

17) Wang HL, Carroll MJ. Guided bone regeneration using bone grafts and collagen membranes. Quintessence Int 2001; 32 : 504-515.

18) Jianqi H, Hong H, Lieping S, Genghua G. Comparison of calcium alginate film with collagen membrane for guided bone regeneration in mandibular defects in rabbits. J Oral Maxillofac Surg 2002; 60: 1449-1454.

19) Miyahara T, Nyan M, Shimoda A, Yamamoto Y, Kuroda S, Shiota M, et al. Exploitation of a novel polysaccharide nanogel cross-linking membrane for guided bone regeneration (GBR). J Tissue Eng Regen Med 2012; 6: 666-672.

20) Zhao S, Pinholt EM, Madsen JE, Donath K. Histological evaluation of different biodegradable and non-biodegradable membranes implanted subcutaneously in rats. J Craniomaxillofac Surg 2000; 28: 116-122.
21) Bunyaratavej P, Wang HL. Collagen membranes: a review. J Periodontol 2001; 72: 215-229.

22) Lynn AK, Yannas IV, Bonfield W. Antigenicity and immunogenicity of collagen. J Biomed Mater Res B Appl Biomater 2004; 71: 343-354.

23) Waksman R. Update on bioabsorbable stents: from bench to clinical. J Interv Cardiol 2006; 19: 414-421.

24) Ueki K, Okabe K, Miyazaki M, Mukozawa A, Moroi A, Marukawa K, et al. Skeletal stability after mandibular setback surgery: Comparisons among unsintered hydroxyapatite/ poly-L-lactic acid plate, poly-Llactic acid plate, and titanium plate. J Oral Maxillofac Surg 2011; 69: 1464-1468.

25) Shikinami Y, Okuno M. Bioresorbable devices made of forged composites of hydroxyapatite (HA) particles and poly-Llactide (PLLA): Part I. Basic characteristics. Biomaterials 1999; 20: 859-877.

26) Shikinami Y, Matsusue Y, Nakamura T. The complete process of bioresorption and bone replacement using devices made of forged composites of raw hydroxyapatite particles/ poly l-lactide (F-u-HA/PLLA). Biomaterials 2005; 26: 5542 5551.

27) Ueki K, Marukawa K, Moroi A, Sotobori M, Ishihara Y, Iguchi $\mathrm{R}$, et al. Evaluation of overlapped cortical bone area after modified plate fixation with bent plate in sagittal split ramus osteotomy. J Craniomaxillofac Surg 2014; 42: e210-e216.

28) Ikawa H, Moroi A, Yoshizawa K, Saida Y, Hotta A, Tsutsui $\mathrm{T}$, et al. Bone regeneration enhancement by ultra-violet (UV) treatment for uHA/PLLA absorbable mesh. J Craniomaxillofac Surg 2017; 45: 634-641.

29) Moroi A, Okuno M, Kobayashi G, Gamo H, Serizawa I, Yoshizawa K, et al. Effect on surface character and mechanical property of unsintered hydroxyapatite/poly-l-lactic acid (uHA/PLLA) material by UV treatment. J Biomed Mater Res B Appl Biomater 2018; 106: 191-200.

30) Retzepi M, Donos N. Guided bone regeneration: biological principle and therapeutic applications. Clin Oral Implants Res 2010; 21: 567-576.

31) Medeiros ES, Glenn GM, Klamczynski AP, Orts WJ, Mattoso LHC. Solution blow spinning: a new method to produce micro-and nanofibers from polymer solutions. J Appl Polym Sci 2009; 113: 2322-2330.

32) Sakaguchi K, Morita I. Relationship between the ablility to support differentiation of osteoclast-like cells and adipogenesis in murine stromal cells derived from bone marrow. Prostaglandins Leukot Essent Acids 2000; 62: 319327.

33) Terranova L, Mallet R, Perrot R, Chappard D. Polystyrene scaffolds based on microfibers as a bone substitute; development and in vitro study. Acta Biomater 2016; 29: 380388.

34) Kusumoto Y, Tachikawa N, Munakata M, Miyahara T, Kasugai S. Lateral bone window closing technique with polyL-lactic acid (PLLA) membrane in the augmentation of the maxillary sinus without grafting material: evaluation of bone healing in a rabbit model. Clin Implant Dent Relat Res 2016; 18: 261-269. 